University of Nebraska - Lincoln

DigitalCommons@University of Nebraska - Lincoln

December 1971

\title{
Shubnikov-de Haas Effect and Magnetic Breakdown in Be
}

\author{
David J. Sellmyer \\ University of Nebraska-Lincoln, dsellmyer@unl.edu
}

\section{I.S. Goldstein}

Center for Materials Science and Engineering and Department of Metallurgy and Materials Science, Massachusetts Institute of Technology, Cambridge, Massachusetts

\section{B.L. Averbach}

Center for Materials Science and Engineering and Department of Metallurgy and Materials Science, Massachusetts Institute of Technology, Cambridge, Massachusetts

Follow this and additional works at: https://digitalcommons.unl.edu/physicssellmyer

Part of the Physics Commons

Sellmyer, David J.; Goldstein, I.S.; and Averbach, B.L., "Shubnikov-de Haas Effect and Magnetic Breakdown in Be " (1971). David Sellmyer Publications. 180.

https://digitalcommons.unl.edu/physicssellmyer/180

This Article is brought to you for free and open access by the Research Papers in Physics and Astronomy at DigitalCommons@University of Nebraska - Lincoln. It has been accepted for inclusion in David Sellmyer Publications by an authorized administrator of DigitalCommons@University of Nebraska - Lincoln. 


\title{
COMMENTS AND ADDENDA
}

The Comments and Addenda section is for short communications which are not of such urgency as to justify publication in Physical Review Letters and are not appropriate for regular Articles. It includes only the following types of communications: (1) comments on papers previously published in The Physical Review or Physical Review Letters; (2) addenda to papers previously published in The Physical Review or Physical Review Letters, in which the additional information can be presented without the need for writing a complete article. Manuscripts intended for this section may be accompanied by a brief abstract for information-retrieval purposes. Accepted manuscripts will follow the same publication schedule as articles in this journal, and galleys will be sent to authors.

\section{Shubnikov-de Haas Effect and Magnetic Breakdown in $\mathrm{Be}^{\dagger}$}

\author{
D. J. Sellmyer, I. S. Goldstein, ${ }^{*}$ and B. L. Averbach \\ Center for Materials Science and Engineering and Department of Metallurgy and Materials Science, \\ Massachusetts Institute of Technology, Cambridge, Massachusetts 02139 \\ (Received 6 July 1971)
}

\begin{abstract}
Magnetic breakdown in the basal plane of Be was investigated in fields up to $150 \mathrm{kG}$. Geometrical aspects of the open orbits caused by breakdown a re discussed. An estimate of $30 \mathrm{kG}$ for the characteristic breakdown field is obtained by applying the theory of Falicov et al. Shubnikov-de Haas frequency measurements are reported for the central-cigar cross section. These measurements are relevant to a recent conjecture that the Onsager-Lifshitz quantization scheme may be violated in the presence of magnetic breakdown.
\end{abstract}

The phenomenon of magnetic breakdown in metals has been investigated experimentally and theoretically, particularly in hexagonal-close-packed metals such as $\mathrm{Zn}$ and $\mathrm{Mg}$. ${ }^{1,2}$ The purpose of the present work was to investigate this phenomenon in $\mathrm{Be}$ and to compare high-field-magnetoresistance measurements with the theory of Falicov et al. ${ }^{2}$ Their calculation of the transverse magnetoresistance of $\mathrm{Zn}$ and $\mathrm{Mg}$ is applicable to Be because the geometry of the pieces of Fermi surface involved in the breakdown is similar in all three metals. ${ }^{2,3}$ The existence of breakdown in the basal plane in $\mathrm{Be}$ has been shown by Alekseevskii and Egorov, ${ }^{4}$ Reed, ${ }^{5,6}$ and Reed and Condon. ${ }^{7}$ However, these authors did not attempt a quantitative comparison of their results with theory. Another motivation for this work was to investigate further the conjecture of Alekseevskii and Egorov ${ }^{4}$ that the Onsager-Lifshitz quantization scheme may be violated, in the presence of magnetic breakdown, in such a way that different frequencies could be observed in the Shubnikov-de Haas (SdH) and de Haas-van Alphen (dHvA) effects.

The measurements were made at the Francis Bitter National Magnet Laboratory in fields up to $150 \mathrm{kG}$ and at $T=4.2^{\circ} \mathrm{K}$. Measurements below 4.2 ${ }^{\circ} \mathrm{K}$ were not performed because magnetic-interaction effects become predominant there as reported by Reed and Condon. ${ }^{7}$ The electronic techniques and sample holder were those described previously. ${ }^{8,9}$ The sample, obtained from the Franklin Institute, was a single-crystal rod whose current density $\vec{J}$ was parallel to $[1 \overline{12} 0]$; its residual-resistance ratio $\left[\rho\left(296{ }^{\circ} \mathrm{K}\right) / \rho\left(4.2^{\circ} \mathrm{K}\right)\right]$ was $100 .{ }^{10}$

The effects of magnetic breakdown are demonstrated by the longitudinal-to-transverse magnetoresistance plot of Fig. 1. The field is in the (1100) plane and open orbits induced by breakdown are shown by the dip centered on [0001]. The orbits are directed along [1100] and exist for angles of $\vec{B}$ out to $19.2^{\circ} \pm 0.8^{\circ}$ away from [0001]. This estimate, obtained from the width of the dip at half-amplitude, agrees with the value of $19.5^{\circ}$ given by Reed. ${ }^{6}$ The geometry of the open orbit caused by breakdown between the coronet and cigars is shown in Fig. 2. This figure was constructed, approximately to scale, from the work of Tripp et al. ${ }^{3}$ The geometrical factors that control the thickness of the band of open orbits, as the field is tipped away from [0001], are the size of the neck orbit on the coronet and the distance along the hexad axis over which breakdown occurs on the cigar. At a critical angle $\psi$, corresponding to $\sim 19.2^{\circ}$, the thickness of the open-orbit band approaches zero as shown in Fig. 2. The implication of this value is that magnetic breakdown occurs over a length $2 l \simeq 0.063$ a.u., which is approximately $7 \%$ of the length of the cigar. Since the energy gap increases 


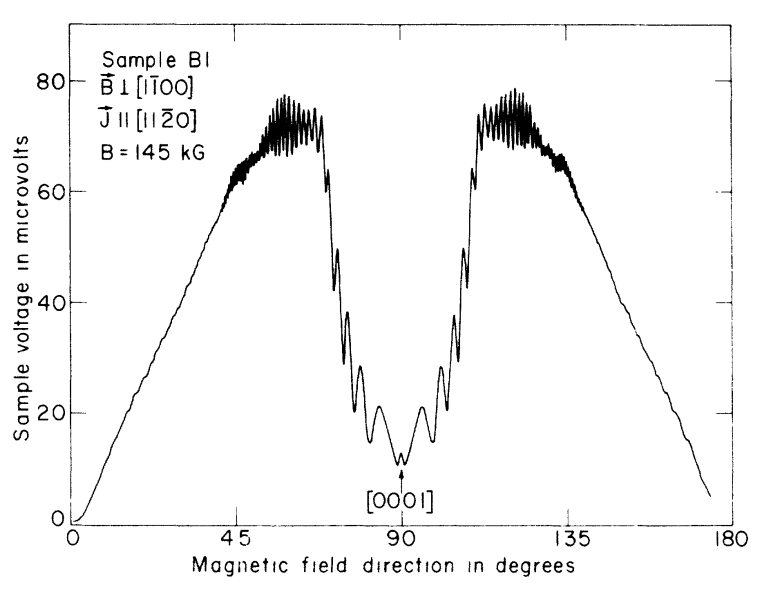

FIG. 1. Longitudinal-to-transverse magnetoresistancerotation plot at $145 \mathrm{kG}$. The sample notation is that of Ref. 10.

with distance along the hexad axis, the breakdown length would be expected to increase with field above the minimum breakdown field. Thus the above breakdown length is that corresponding to a field of $145 \mathrm{kG}$.

Figure 1 shows also some rather large-amplitude quantum oscillations which are due to breakdown. These oscillations show up clearly in field sweeps for $\vec{B}$ near [0001], where their amplitude is $\sim 50 \%$ of the dc-background magnetoresistance. The $\mathrm{SdH}$ frequency of the oscillations at $[0001]$ is $9.4 \times 10^{6}$

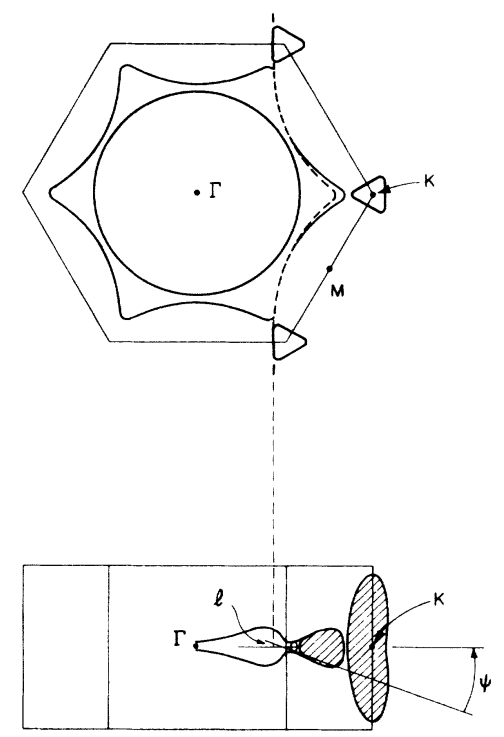

FIG. 2. Fermi-surface model of Be showing the secondzone coronet and third-zone cigars. The open orbit at the critical angle $\psi$ is shown in dashed lines in the upper view. The model is based on the work of Tripp et al. (Ref. 3).

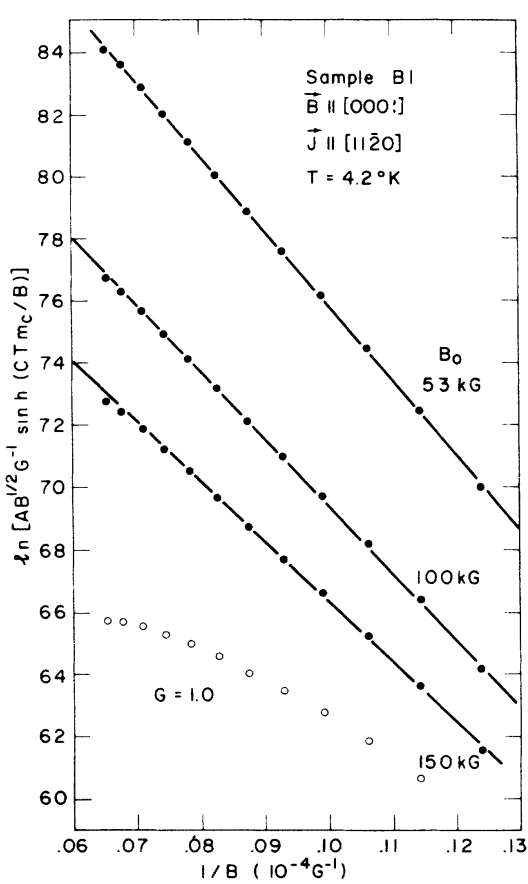

F IG. 3. Scattering-temperature plot showing the effect of breakdown through the probability $\mathrm{G}$ for completing a complete cigar orbit.

G, which corresponds to the central-cigar cross section. Figure 3 shows the amplitude of the oscillations, as a function of $B^{-1}$, on the usual scattering-temperature plot. ${ }^{11}$ The circles are the experi-

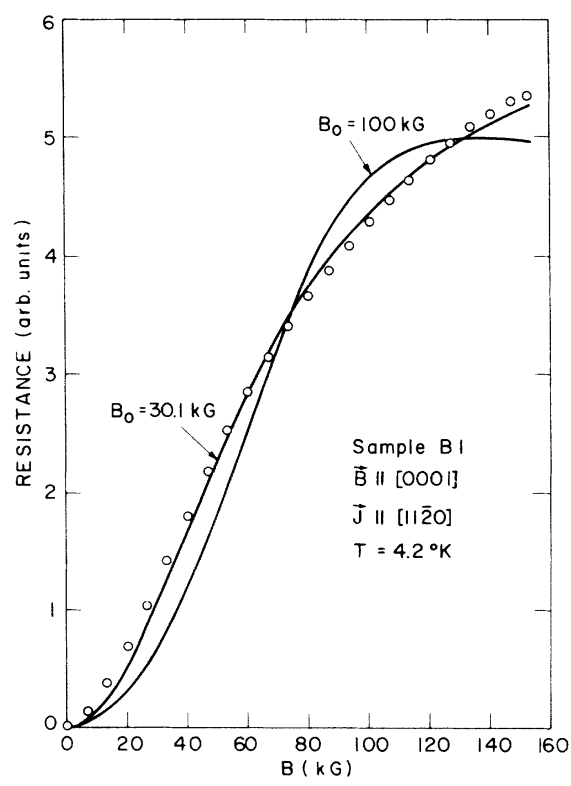

FIG. 4. Experimental data (open circles) and fits of the theory of Falicov et al. (Ref. 2) to the nonoscillatory magnetoresistance. Fits with two values of $B_{0}$ are shown. 
mental points and the dots were obtained by correcting the data by a factor $G \equiv\left(1-e^{-B_{0} / B}\right)^{3 / 2}$, which is the probability that an electron completes a cyclotron orbit on the cigar without breakdown. ${ }^{2}$ Three values of the characteristic breakdown field $B_{0}$ were tried, all of order $10^{5} \mathrm{G}$. These values were chosen because (a) the magnetoresistance deviates markedly from quadratic above $\sim 50 \mathrm{kG}$, (b) Alekseevskii and Egorov estimated $B_{0} \sim 150 \mathrm{kG}$ from magnetoresistance measurements, ${ }^{4}$ and (c) Tripp et al. ${ }^{3}$ estimated $B_{0}=120 \mathrm{kG}$ from their pseudopotential calculation. However, as shown in Fig. 3, the quality of the fit is very insensitive to the value of $B_{0}$, so that this method appears to be a poor one for estimating this field. A similar behavior was found by Falicov et al. , ${ }^{2}$ who fitted dHvA amplitude data in Zn essentially equally as well with $B_{0}$ values of 2.7 or $6 \mathrm{kG}$.

In view of this problem an attempt was made to fit the field dependence of the nonoscillatory part of the magnetoresistance, using the theory of Falicov et al. ${ }^{2}$ This theory for $\mathrm{Zn}$ and $\mathrm{Mg}$ divided the conductivity into a classical part for the large orbits in the second zone plus a quantum-mechanical part responsible for the switching probabilities, at the junctions in the third zone, of the classical network. It is assumed that the impurity-scattering probability on the third-zone orbits is so small that it can be neglected in comparison with the probability for interband transitions due to magnetic breakdown. While this assumption is only of marginal validity in our sample, application of the theory to our sample can be justified to some extent since $\left(\omega_{c}^{\prime} \tau\right)_{\text {cigar }} \gg 1$ at the highest fields, ${ }^{10}$ and also because the amplitude of the oscillations $\left(\sim 0.5 \rho_{\text {nonosc }}\right)$ is large enough to indicate that the switching probabilities play a major role in the field dependence. The nonoscillatory part of the magnetoresistance is shown by the points in Fig. 4 . Equation (3.7) of Falicov et al. ${ }^{2}$ was used, along with $^{1}$

$$
\rho_{11}=\sigma_{11}\left(\sigma_{11}^{2}+\sigma_{12}^{2}\right)^{-1},
$$

to fit the field dependence of the magnetoresistance. The full expression for $\rho_{11}$ contained two parameters, in addition to the breakdown field $B_{0}$ to be deter mined. Various values of $B_{0}$ were chosen and a nonlinearleast-squares fit was performed to find the best values of the other parameters. The best fit of the data was obtained with $B_{0}=30.1 \mathrm{kG}$ as shown in Fig. 4. In order to check this fitting procedure for sensitivity to $B_{0}$, the fit obtained with $B_{0}=100 \mathrm{kG}$ is also shown; it is clearly inferior to the $B_{0}=30.1$ $\mathrm{kG}$ fit. It is interesting to note that in the recent work of Reed and Condon 7 on much "higher" purity samples than ours, the effects of magnetic breakdown are already quite obvious at $B \sim 20 \mathrm{kG}$. Thus our estimate of $B_{0} \simeq 30 \mathrm{kG}$ as well as this latter work suggests that $B_{0}$ for $\mathrm{Be}$ is considerably less than earlier estimates of $\sim 150 \mathrm{kG}$. ${ }^{4}$ For a more detailed comparison with theory, measurements of $\rho_{11}$ and $\rho_{12}$, on a sample of higher purity (e.g. , that of Ref. 7), would be desirable.

A final point concerns the result of Alekseevskii and Egorov, ${ }^{4}$ who found that their $\mathrm{SdH}$ frequency results deviated from the dHvA results of Watts by $\sim 15 \%$, which was more than the estimated experimental uncertainties. On this basis they speculated that: "It is not excluded that under the conditions of magnetic breakdown the Lifshitz-Onsager quantization scheme may be violated and the electron motion, strictly speaking, will not be described by the orbits observed in the dHvA effect." Figure 5 shows our $\mathrm{SdH}$ frequency data $[F(\theta)]$ in the $(1 \overline{1} 00)$ plane, along with the dHvA data of Tripp et al. ${ }^{3}$ $F(\theta)$ was obtained from the data of Fig. 1, using the constant-field rotation method. Our results show no discrepancy with the dHvA results of Tripp et al. ${ }^{3}$ Except perhaps for systematic frequencycalibration or sample-orientation errors, it is difficult to understand the source of the disagreement in the earlier work. Since the frequency is controlled by geometrical factors alone, it is reasonable that the central-cigar frequency is the same in both the dHvA and SdH effects.

In summary, the open-orbit region at $145 \mathrm{kG}$ was estimated as approximately $7 \%$ of the length of the third-zone cigars. This estimate relied heavily on the Fermi-surface model of Tripp et al. ${ }^{3}$ It was shown that the simple method of applying a correction factor for breakdown to the SdH amplitude data was a poor method for estimating the breakdown field. However, a fit of the nonoscillatory part of the magnetoresistance to the theory of Falicov et $a l .^{2}$ gave $B_{0} \simeq 30 \mathrm{kG}$. This value appears to be more

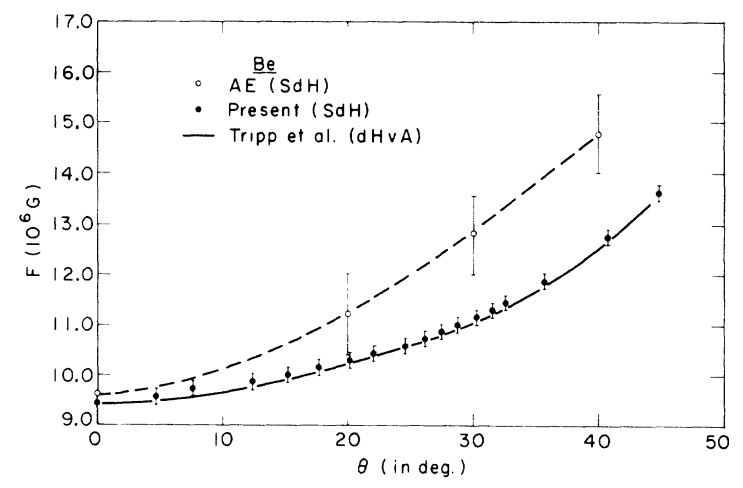

FIG. 5. Comparison of the SdH frequency data with those of Alekseevskii and Egorov (AE) (Ref. 4), and with the dHvA data of Tripp et al. (Ref. 3). 
reasonable, when compared with the recent data of Reed and Condon, ${ }^{7}$ than earlier estimates of $B_{0}$ $\geq 100 \mathrm{kG}$. Finally, the orientation dependence of the $\mathrm{SdH}$ frequency was found to agree well with $\mathrm{dHvA}$ results, in contradiction to recent results in the literature.

\footnotetext{
$\dagger$ Research supported by the National Science Foundation, Advanced Research Projects Agency, and U. S. Air Force Materials Laboratory, Wright-Patterson Air Force Base, Ohio. The work was performed at the Francis Bitter National Magnet Laboratory, Cambridge, Mass., while it was supported by the U. S. Air Force Office of Scientific Research.

${ }^{*}$ Present address: Xerox Corporation, Rochester, N. Y.

${ }^{1}$ See R. W. Stark and L. M. Falicov, in Progress in Low Temperature Physics, edited by C. J. Gorter (NorthHolland, Amsterdam, 1967), Vol. V, p. 235, and references therein.

${ }^{2}$ See L. M. Falicov, A. B. Pippard, and P. R. Sievert, Phys. Rev. 151, 499 (1966), and references therein.

${ }^{3}$ J. H. Tripp, P. M. Everett, W. L. Gordon, and R. W. Stark, Phys. Rev. 180,669 (1969).

${ }^{4}$ N. E. Alekseevskii and V. S. Egorov, Zh. Eksperim.
}

i Teor. Fiz. $\underline{55}, 1153$ (1968) [Sov. Phys. JETP $\underline{28}, 601$ (1969) ], and references therein.

${ }^{5}$ W. A. Reed, Bull. Am. Phys. Soc. 9, 633 (1964).

${ }^{6} \mathrm{~W}$. A. Reed, in Proceedings of the Eleventh International Conference on Low-Temperature Physics, edited by J. F. Allen (University of St. Andrews Printing Department, St. Andrews, Scotland, 1969), p. 1160.

${ }^{7}$ W. A. Reed and J. H. Condon, Phys. Rev. B 1,3504 (1970).

${ }^{8}$ D. J. Sellmyer, Rev. Sci. Instr. 38,434 (1967).

${ }^{9}$ D. J. Sellmyer, J. Phys. Chem. Solids 30,2371 (1969).

${ }^{10}$ I. S. Goldstein, D. J. Sellmyer, and B. L. Averbach, Phys. Rev. B 2,1422 (1970).

${ }^{11}$ I. M. Lifshitz and A. M. Kosevich, Zh. Eksperim. i Teor. Fiz. 29, 730 (1956) [Sov. Phys. JETP $\underline{2}, 636$ (1956)].

\title{
Comments on Pseudopotential Form Factors for White Tin
}

\author{
M. A. C. Devillers and A. R. de Vroomen \\ Fysisch Laboratorium, Katholieke Universiteit, Nijmegen, The Netherlanas \\ (Received 19 July 1971)
}

While preparing for an experimental investigation of electron-phonon mass enhancement and relaxation times in white tin, we were confronted with large inconsistencies in the published form factors for this metal. ${ }^{1-5}$ In this paper we are presenting the results of calculations that should clarify this matter a great deal.

The experiments on which our calculations are based are Fermi-surface calipers obtained from very accurate $\mathrm{rf}$ size-effect measurements. ${ }^{6}$ With a plane-wave matrix, large enough to assure convergence with respect to the number of reciprocal lattice vectors, we fitted the four form factors $V\left(K_{n}\right), K_{n}<2 k_{F}$, and the Fermi energy (or mass) at 14 selected points, representative of the Fermi surface. The best fit is obtained for $E_{F}=2.11$ (throughout this paper, we use $h^{2} / 2 m a^{2}$ as unit of energy) [Fig. 1(a)]. Compared with the nearly-freeelectron value $E_{\mathrm{NFE}}=2.312$, this suggests a Fermisurface-band effective mass of about $0.92 m_{0}$. For the best energy value the fit shows a rms deviation in the calipers of about $0.004 k_{0}\left(k_{0}=\Gamma L=2 \pi / a\right)$, which is slightly larger than the experimental inaccuracies. In Fig. 1(a) one sees that this Fermi energy can be determined to within 0.01 (our units). Our fitted form factors show a large energy de- pendence [Fig. 1(b)]. We are confident that these curves are representative of the entire Fermi surface, rather than of the specific sample of data points.

In the following we shall compare our results with previous calculations. The work of Weis $z^{1}$ was based on Gantmakher's pioneering rf sizeeffect data. Due to some uncertainties in the line shape the inaccuracy of Gantmakher's calipers amounts to several percent. Weisz found a best Fermi energy of about 2.24 (our units). For this energy we find a fit which has a rms deviation seven times larger than that of our best fit. We feel that Weisz's deviation from our results is due to the large limitation in accuracy and in the number of his data points.

Stafleu and de Vroomen ${ }^{2}$ took $E_{F}$ equal to the freeelectron value and used a "few-plane-wave" matrix. In view of their aim to obtain a rough agreement with their de Haas-van Alphen (dHvA) experiments the discrepancies with our results are reasonable.

Craven's results ${ }^{3}$ are of more serious concern to us. He claims to describe to within $1 \%$ the accurate dHvA measurements of Craven and Stark ${ }^{7}$ by form factors, given in Fig. 1(b), at an energy $E_{F}=2.34$ (our units). However, his interpolated 